\title{
Call for Alleviating Sexual Issues among Cervical Cancer Survivors' Women in Northern Upper Egypt
}

\author{
Hanan Elzeblawy Hassan ${ }^{1}$, Ragaa Ali Mohammed ${ }^{2}$, Soad Abd El Salam Ramadan ${ }^{3}$, Hagar kamal masaud ${ }^{4}$ \\ ${ }^{1}$ Associate professor Maternal and Newborn Health Nursing, Faculty of Nursing, Beni-Suef University, Egypt \\ ${ }^{2}$ Professor of Maternity \& Neonatal Nursing, Faculty of Nursing, Cairo University, Egypt \\ ${ }^{3}$ Professor of Obstetrics and Women Health Nursing, Faculty of Nursing, Benha University, Egypt \\ ${ }^{4}$ Assistant lecturer of Maternal and Newborn Health Nursing, Faculty of Nursing, Beni-Suef University, Egypt
}

*Corresponding author: Hanan Elzeblawy, Associate professor Maternal and Newborn Health Nursing, Faculty of Nursing, Beni-Suef University, Egypt.

Received date: March 11, 2021; Accepted date: March 20, 2021; Published date: March 29,2021

Citation: Hanan E. Hassan, Ragaa A. Mohammed, Soad A. S. Ramadan, Hagar K. masaud (2021) Call for Alleviating Sexual Issues among Cervical Cancer Survivors' Women in Northern Upper Egypt. J. Obstetrics Gynecology and Reproductive Sciences 5(3); DOI: 10.31579/2578-8965/066

Copyright: (C) 2021, Hanan Elzeblawy, This is an open access article distributed under the Creative Commons Attribution License, which permits unrestricted use, distribution, and reproduction in any medium, provided the original work is properly cited.

\section{Abstract}

Background: Cervical cancer affects all aspects of a patient's life, including sexual functioning, body image, and intimacy.

Aim: The present study aimed to evaluate the impact of a protocol of nursing intervention on sexual dysfunction and body image among cervical cancer survivors' women.

Methods; Design: A quasi-experimental research design was conducted. Setting: out-patient clinic in the oncology unit at Beni-Suef University Hospital. Subjects: A purposive sample of 70 women. Tools: Data was collected through a structured interviewing questionnaire sheet, female sexual function index, female sexual distress scale, and body image scale.

Results: The results of the study revealed that there was a high statistically significant difference in the women's total score of knowledge about cervical cancer, total scores of female sexual function index, female sexual distress scale, and body image scale at post-intervention compared to pre-intervention $(\mathrm{p}<0.001)$.

Conclusion: The study concluded that educational protocol, which provided to cervical cancer survivors' women, have a positive effect on their own knowledge, body image, and sexual function.

Recommendations: disseminate the multidisciplinary collaboration approach for addressing sexual problems related to cervical cancer, and preparing health classes for cervical cancer women regarding sexual dysfunction with cervical cancer.

Keywords: cervical cancer, sexual dysfunction, sexual distress, body image

\section{Introduction:}

Cancer is increasingly growing as a major public health problem in both developed and developing countries. Cancer can impose health, heavy economic and social burden. It is a global pandemic affecting both developed and developing regions, but it is rapidly increasing in low and middle-income-countries, where resources for prevention, diagnosis, and treatment are limited or non-existent. [1-2]

Cervical cancer (CC) is a health problem due to the high prevalence rates and mortality in women of low socio-economical levels and in the productive phase of their lives. There is a strong association of this type of cancer with precarious living conditions, low indices of human development, the absence or weakness of community education strategies, and the difficulty of access to public health services for the early diagnosis and treatment. [3-5]
Cervical cancer occurs when the cells of the cervix grow abnormally and invade other tissues and organs of the body. The slow-growing of cervical cancer and progression through precancerous changes provides opportunities for prevention, early detection, and treatment. [6-7]

Receiving any cancer diagnosis is an unpleasant experience, accompanied by distress, which impacts a person's personal and family life. [8] As a result of the diagnosis, patients experience socio-economic problems, marital issues, and psychological problems. Intense psychological distress is common not only upon receiving a diagnosis but also throughout treatment. [9-11]

Human papillomavirus (HPV) infection is known as one of the steps of the carcinogenesis process. The infection is more frequent among women in the years following the first sexual intercourse. Precursor lesions can 
occur as a consequence of a persistent infection in a process that lasts for 5 to 10 years. [12]

There are different types of treatment options for patients with cervical cancer. Five types of standard treatment are used: surgery, radiation therapy, chemotherapy, targeted therapy, and immunotherapy. New types of treatment are being tested in clinical trials. Treatment for cervical cancer may cause side effects. Patients may want to think about taking part in a clinical trial. [13]

There is a tendency of reduction or stability in cervical cancer mortality rate and an increase in the number of women living with the healing or chronicity of the disease. However, the increasing survival, an achievement for the oncology area caused by progress in treatment, is permeated by these treatment consequences, which persist for years. [14] The effects of therapies and the disease adversely affect the quality of life and sexual function of cervical cancer surviving patients. The various therapeutic modalities interfere with the functioning of the pelvic organs, causing sexual, urinary, and intestinal dysfunction. [15]

Sexual dysfunction is one of the most distressful symptoms among cervical cancer survivors. Cancer treatment including radiotherapy results in a high degree of vaginal morbidity and persistent sexual dysfunction. The vaginal symptoms reported after cervical cancer treatment, include sore membranes, reduced lubrication, and genital swelling which severely affect the women's sexual health. [16-19]

Nurses play an essential role in healthcare systems, representing a significant proportion of the entire healthcare workforce. About 23 million nurses work in the healthcare sector globally [20-26]. Maternity nurses play a crucial role in the quality of care improvement, which provides women education and support in multi-dispelling areas. At the same time, the nurse can provide health promotion \& psychosocial services include assessment, health education, counseling \& appropriate referral [27-36].

The nurse is an important member of the health care team, to counsel women in the sensitive and highly charged area of human sexuality. Sexuality and sexual health problems are challenging areas for nurses so should be approached in a way that respects women's confidentiality and sensitively explores women's needs. [37-39] Nursing interventions (Education and counseling on sexuality) are used to assist women to resolve their sexual problems. [38]

Education and counseling on sexuality are nursing interventions used to assist patients to resolve their sexual problems. In nurse-led counseling, a nurse provides information and assists patients in making and executing a decision; the nurse also guides the cervical cancer survivor to regain self-confidence and adapt to physical and psychological changes to optimize survivor autonomy. Nurse-led psychosexual counseling can significantly improve sexual function in patients with gynecological cancer. [8, 37] Education and counseling for women after cancer treatment may also reduce sexual problems and improve the marital relationship. $[8,40]$

\section{Significance of the study:}

Sexual oncology is gaining appreciation as a major area needing attention in nursing practice and research. Oncology nurses need to possess a high level of sensibility in dealing with women's sexual health needs. However, sexual health care is still inadequately addressed due to barriers such as incorrect assumptions and beliefs toward sexual issues. [41] One of the main roles of oncology nurses is to assess problems in this area to be able to provide anticipatory guidance related to treatment and the resumption of sexual activity, but this is one aspect of care that has been largely ignored by health care providers. [42-45] Sexuality issues have not been adequately addressed by health care providers. [37]

\section{Aim of the study:}

The aim of this study is to designing, implement, and evaluating the impact of the application of protocol of nursing intervention on sexual dysfunction (sexual distress, sexual functions) and body image among cervical cancer survivors' women.

\section{Hypothesis}

Cervical cancer survivors' women that adopt the protocol of nursing intervention will experience regression in sexual distress and progression in sexual function and body image, as well.

\section{Subjects and methods:}

\section{Research Design}

The study followed a quasi-experimental (pre-post) test study design.

\section{Setting}

The study was conducted in an out-patient clinic in the oncology unit at Beni-Suef University hospital.

\section{Subjects:}

Type: a purposive sample of women who met the criteria for inclusion in this study.

Size: 70 women who fulfilled inclusion criteria were selected. Steven and Thompson's equation was used to calculate the sample size from the next formula;

$$
n=\frac{N p(1-P)}{(N-1)\left(\frac{d^{2}}{z^{2}}\right)+P(1-P)}
$$

$\mathrm{N}=$ Population (140)

$\mathrm{Z}=$ confidence level $95 \%(1.96)$

$\mathrm{P}=$ probability $(10 \%)$

$\mathrm{d}=$ margin of error $(0.05)$

Inclusion criteria

All married women that were diagnosed with cervical cancer

Undergoing different types of treatment

With any cervical cancer degree of all ages was recruited.

\section{Exclusion criteria}

Gynecological tumors, e.g. vaginal, breast, and uterine cancer were excluded.

\section{Tools of Data Collection}

To attain the aim of this study, four tools were used for data collection;

Tool I: Structured interviewing questionnaire sheet was developed by the researchers in the Arabic language based on a review of recent literature. It was consisting of three parts:

Part 1: Socio-demographic characteristics of women as age, level of education, occupation, and residence.

Part 2: Medical \& surgical history: how cervical cancer was detected, stage of cervical cancer, type of treatment regimen, types of surgery performed.

Part 3: Women's knowledge about cervical cancer. This part was designed to assess women's knowledge about cervical cancer such as (definition, causes, signs, symptoms, risk factors, methods of prevention, diagnosis, treatment) of cervical cancer. 
Tool II: Female Sexual Function Index (FSFI). A multidimensional self-report questionnaire that assesses the key dimensions of female sexual function during the four weeks before the interview day. For the present study, the researchers will use the Arabic version of FSFI that was translated by (Anis et al., 2011). It was validated for the Egyptian population. [46]

Tool III: Female sexual distress scale; it revised for assessing distress in women with hypoactive sexual desire disorder. This is a self-report questionnaire designed by Derogatis et al., 2008). [47]

Tool V: Body Image Scale; It is a self-report measure of the woman's body image. This scale was constructed in collaboration with the European Organization for Research and Treatment of Cancer (EORTC) designed by Hopwood et al, 2001. [48]

\section{Validity and Reliability:}

Content validity of the questionnaire was ensured through 3 experts in maternity/obstetrics/gynecological nursing. Modification to the tools was made according to experts' judgment on the clarity of sentences, appropriateness of the content, the sequence of items, and accuracy of scoring and recording of items. Cronbâch alpha and Spearman-Brown coefficients were calculated to assess the reliability of the developed tools through their internal consistency.

\section{Administrative \& Ethical Considerations:}

Before conducting the study, official permission was obtained from the director of Beni-Suef University Hospitals. Consent was obtained from each woman recruited in the study. Participants' were told that all their data were highly confidential. Each woman had the right to withdraw from the study at any time, confidentiality was maintained and human rights were used.

\section{Field work:}

\subsubsection{Preparatory phase:}

It was included reviewing the national and international related literature, and theoretical knowledge about various aspects of the study problem. This helped the researchers to be acquainted with the magnitude of the problems and guided the researcher to prepare the required data collection tools. Then the researcher tested the validity of the tool through a jury of expertise to test the content, knowledge, accuracy \& relevance of questions for tools.

\subsubsection{Pilot study:}

A pilot study was conducted on $10 \%$ (7women) to evaluate the applicability, efficiency, clarity of tools, assessment of the feasibility of fieldwork and identification of the suitable place for interviewing the studied women, besides to detect any possible obstacles that might face the researcher and interfere with data collection. Necessary modifications were done based on the pilot study findings such as (omission of some questions from the tool) to strengthen their contents or for more simplicity and clarity. The pilot sample was excluded from the main study sample.

\subsubsection{Data collection phase (field work phase)}

The data was collected through a period of six months from 1/8/2019 till $31 / 1 / 2021$.

The researcher was attended at the previously mentioned setting till all the pre-mentioned sample size collected. The researcher introduced herself to women and explains the aim of the study before data collection. The sample was collected 2-days per week from 9 am to 2 $\mathrm{pm}$. The approval of women was obtained orally before data collection.

The researcher filled the interviewing questionnaire from the women.
The filling questionnaire ranged from 15 to 20 minutes for each woman. The sexual nursing counseling was given by the researcher at the outpatient unit in three meeting sessions.

An educational booklet was distributed to participant women. It designed by the researchers and used as a supportive material and based on the review of literature containing data regarding Cervical cancer (causes, degrees, treatment, and management of treatments' side effects), Physical activity (walking, range of motion exercise, relaxation techniques, breathing exercise, distraction, and recreation), Diet therapy (high fiber diet, low-fat diet, high vegetables/fruits diet), Information and education on reproductive organs and sexual function (anatomy and physiology of female genital system, explanation in the series of female sexual response cycle), Types of sexual dysfunctions (sexuality problems, numerous relaxation, exercises for improving sexual fitness such as Kegel exercise, sensation focus exercise, and exercise of various technical positions during sexual intercourse).

Weekly follow up by using telephone call for instruction \& reinforcement about items of sexual counseling. The effect of sexual nursing intervention was evaluated by comparing between the women's condition (dysfunction, sexual distress and body image distress) pre and post-intervention after one-month-later.

\section{Statistical analysis}

The collected data were revised, coded, tabulated, and introduced to a PC using a statistical package for social sciences (IBM SPSS 25.0). Data were presented and suitable analysis was done according to the type of data obtained for each parameter

\section{Descriptive Statistics:}

Mean and Standard deviation (SD) and range for parametric numerical data.

Frequency and percentage of non-numerical data.

Analytical Statistics:

Quantitative continuous data were compared using Student t-test in case of comparisons between two independent groups. When a normal distribution of the data could not be assumed, the non-parametric MannWhitney or Kruskal-Wallis tests were used.

Qualitative categorical variables were compared using the Chi-square test. Whenever the expected values in one or more of the cells in a $2 \times 2$ tables was less than 5, Fisher exact test was used instead.

In larger than $2 \times 2$ cross-tables, no test could be applied whenever the expected value in $10 \%$ or more of the cells was less than 5. Spearman rank correlation was used for assessment of the inter-relationships among quantitative variables and ranked ones.

To identify the independent predictors of the knowledge and practice scores multiple linear regression analysis was used and analysis of variance for the full regression models was done. Statistical significance was considered at $\mathrm{p}$-value $<0.05$.

Par and Pie charts were used for graphic presentation.

\section{Results:}

Figure (1) reveals that more than half $(51.4 \%)$ of the study sample their age more than 50 years old. Around one half (48.6\%) of them had secondary education, and more than half $(64.3 \%)$ of women were housewives, and $52.8 \%$ of women were from urban areas

Figure ( 2 \& 3) show that more than one-third (35.7\%) of women were in the $1^{\text {st }}$ degree when diagnosed with cervical cancer while $(4.3 \%)$ were in the $4^{\text {th }}$ degree. Regarding treatment type; only $5.7 \%$ of women 
had received radiotherapy. However, $94.4 \%$ of them treated their cervical cancer by chemotherapy and surgical operation (20.0\%), radiotherapy and surgical operation $(24.3 \%)$, radiotherapy, chemotherapy and surgical operation $(37.1 \%)$, and $12.9 \%$ had managed their cancer surgically.

Table (1) shows that only (4.3\%) of women had satisfactory knowledge during pre-intervention and the majority of them $(95.7 \%)$ had satisfactory knowledge at post-intervention, there was a high statistical significant improvement in the women's total knowledge regarding cervical cancer $(\mathrm{p}<0.001)$.

Table (2) illustrates that all women (100\%) of the studied had sexual dysfunction at pre-intervention phase; while changed to (50\%) at postintervention. A highly statistically significant difference is observed in the total scores of FSFI among the studied sample at pre and post-intervention $(\mathrm{p}<0.001)$.
Table (3) reveals that, however, more than three-quarters $(88.6 \%)$ of the studied women had sexual distress at pre-intervention; all (100\%) of them had no sexual distress at post-intervention. A highly statistically significant difference is observed among women in the total scores of the sexual distress scale $(\mathrm{p}<0.001)$.

Table (4) indicates that more than three-quarters (90\%) of the studied women had body image distress at pre-intervention while nearly all $(97.1 \%)$ of them had good body image at post-intervention. A highly statistically significant difference is observed among women in the total scores of the body image scale $(\mathrm{p}<0.001)$.

Table (5) reveals that there was a correlation between total knowledge scores of women and their total body image scores and there was no correlation between total knowledge scores of women and their total sexual distress scores.

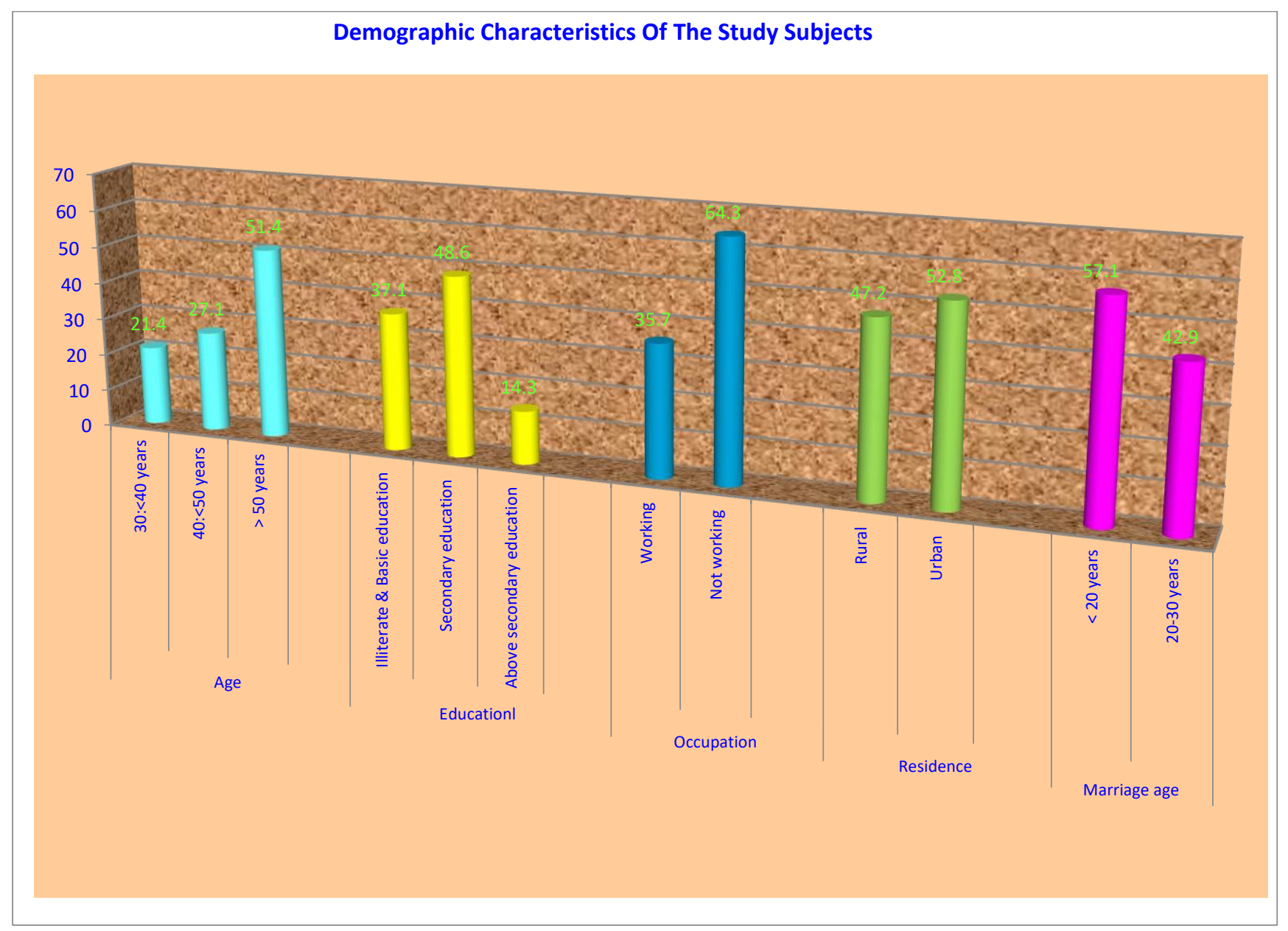

Figure (1): Distribution of demographic characteristics of the study subjects $(n=70)$. 


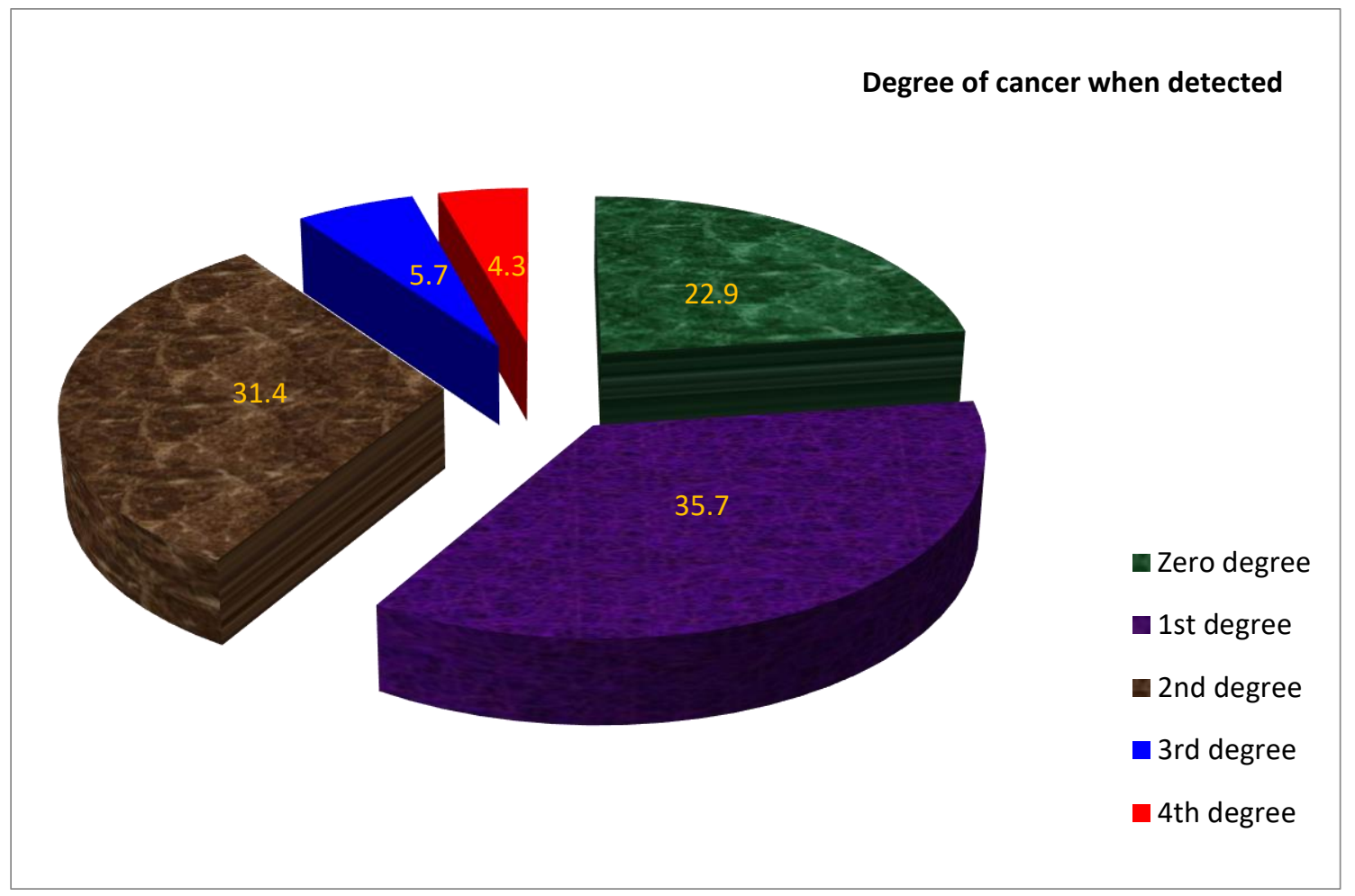

Figure (2): Degree of cervical cancer when detected

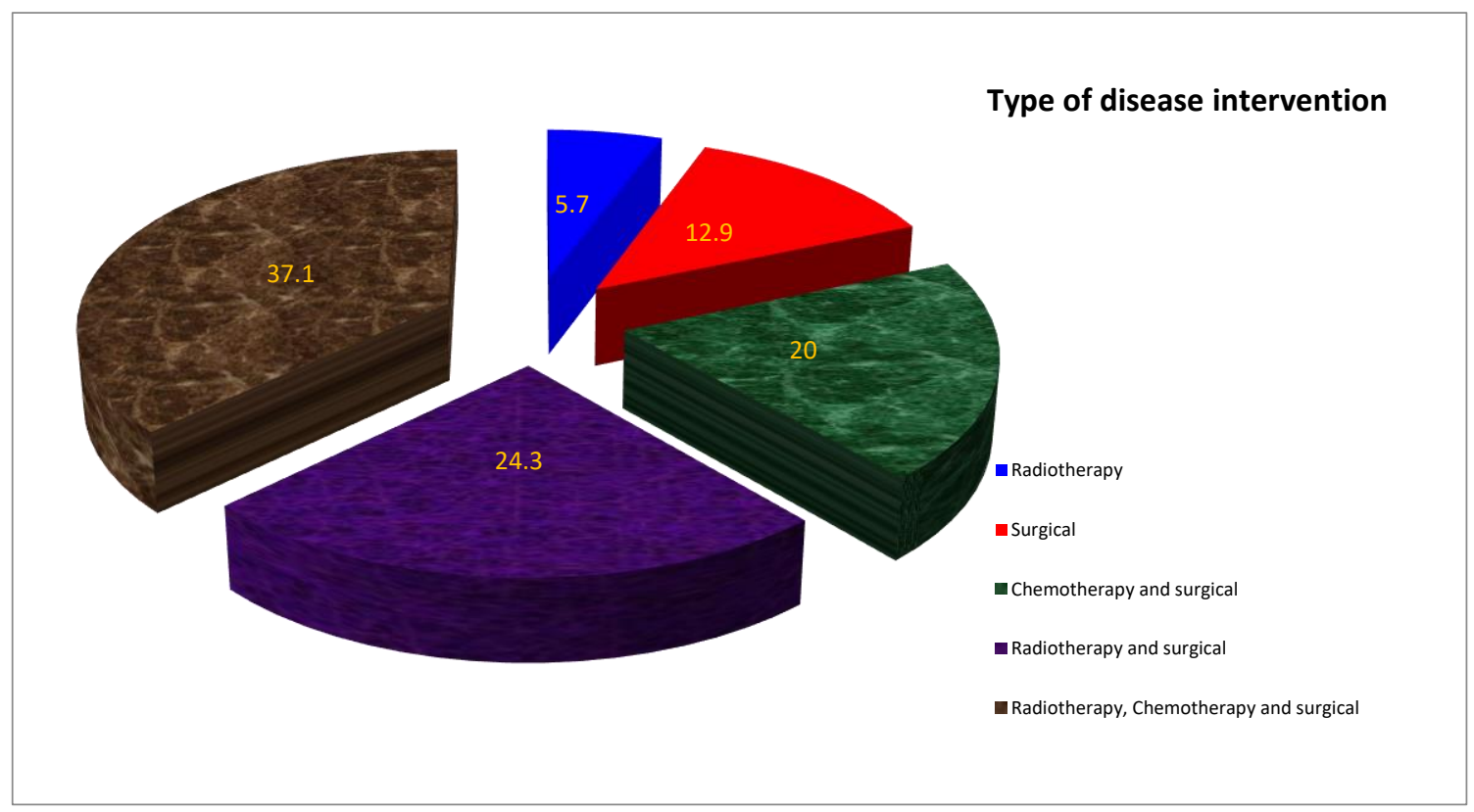

Figure (3): Type of intervention

\begin{tabular}{|c|c|c|c|c|c|c|}
\hline & \multicolumn{2}{|c|}{ Pre-intervention } & \multicolumn{2}{|c|}{ Post-intervention } & \multirow{2}{*}{$\mathbf{X}^{2}$} & \multirow{2}{*}{ P-value } \\
\hline & No. & $\%$ & No. & $\%$ & & \\
\hline Satisfactory knowledge & 3 & 4.3 & 67 & 95.7 & \multirow{2}{*}{27.12} & \multirow{2}{*}{$0.000 * *$} \\
\hline Unsatisfactory knowledge & 67 & 95.7 & 3 & 4.3 & & \\
\hline
\end{tabular}

(**) highly statistically significant at $\mathrm{P}<0.001$ 


\begin{tabular}{|c|c|c|c|c|c|c|}
\hline & \multicolumn{2}{|c|}{ Pre-intervention } & \multicolumn{2}{|c|}{ Post-intervention } & \multirow{2}{*}{$\mathbf{X}^{2}$} & \multirow{2}{*}{ P-value } \\
\hline & No. & $\%$ & No. & $\%$ & & \\
\hline Sexual functioning & 0 & 0 & 35 & 50 & \multirow{3}{*}{19.17} & \multirow{3}{*}{$0.000 * *$} \\
\hline Sexual dysfunction & 70 & 100 & 35 & 50 & & \\
\hline Mean \pm SD & \multicolumn{2}{|c|}{$33.75 \pm 13.12$} & \multicolumn{2}{|c|}{$55.13 \pm 8.31$} & & \\
\hline
\end{tabular}

(**) highly statistically significant at $\mathrm{P}<0.001$

Table (2): Percentage distribution of women's total sexual functions indicators $(n=70)$.

\begin{tabular}{|c|c|c|c|c|c|c|}
\hline & \multicolumn{2}{|c|}{ Pre-intervention } & \multicolumn{2}{|c|}{ Post-intervention } & \multirow{2}{*}{$\mathbf{X}^{2}$} & \multirow{2}{*}{ P-value } \\
\hline & No. & $\%$ & No. & $\%$ & & \\
\hline Sexual distress & 62 & 88.6 & 0 & 0.0 & \multirow{2}{*}{21.46} & \multirow{2}{*}{$0.000 * *$} \\
\hline No sexual distress & 8 & 11.4 & 70 & 100 & & \\
\hline
\end{tabular}

(**) highly statistically significant at $\mathrm{P}<0.001$

Table (3): Percentage distribution of women's total sexual distress scores $(n=70)$.

\begin{tabular}{|l|c|c|c|c|c|c|}
\hline \multirow{2}{*}{} & \multicolumn{2}{|c|}{ Pre-intervention } & \multicolumn{2}{|c|}{ Post-intervention } & \multirow{2}{*}{$\mathbf{X}^{2}$} & \multirow{2}{*}{ P-value } \\
\cline { 2 - 5 } & $\mathbf{N o}$ & $\mathbf{\%}$ & No. & $\boldsymbol{\%}$ & \multirow{2}{*}{19.71} & \multirow{2}{*}{$0.000^{* * *}$} \\
\hline Good image & 7 & 10 & 68 & 97.1 & 2.9 & \\
\hline Image distress & 63 & 90 & 2 & & \\
\hline
\end{tabular}

(**) highly statistically significant at $\mathrm{P}<0.001$

Table (4): Percentage distribution of women's total body image scores $(n=70)$.

\begin{tabular}{|l|c|c|c|c|}
\hline & & Total knowledge & Body image scores & Sexual distress scores \\
\hline \multirow{2}{*}{ Total knowledge } & $\mathbf{r}$ & 1 & -0.252 & -0.086 \\
\cline { 2 - 5 } & $\mathbf{p}$ & - & $0.035^{*}$ & 0.481 \\
\hline \multirow{2}{*}{ Body image scores } & $\mathbf{r}$ & -0.252 & 1 & 0.123 \\
\cline { 2 - 5 } & $\mathbf{p}$ & $0.035^{*}$ & - & 0.311 \\
\hline \multirow{2}{*}{ Sexual distress scores } & $\mathbf{r}$ & -0.086 & 0.123 & 1 \\
\cline { 2 - 5 } & $\mathbf{p}$ & 0.481 & 0.311 & - \\
\hline
\end{tabular}

(*) statistically significant $\mathrm{p}<0.05$

(**) high statistically significant $\mathrm{p}<0.01$

Table (5): Correlation matrix of the study variables

\section{Discussion:}

Sexuality is a vital part of the quality of life and overall health. Cancer's diagnosis can affect many aspects of women's daily routine, including their sexuality. Many gynecological oncology patients have fright and care about the fact that their marriage will be at risk and their sexual relationships with their partner will be influenced due to sexual problems that are caused by cancer and its treatment. [37, 49]

Oncology nurses among health care providers are in the first degree to which women can easily explain themselves and can be effective in removing their concerns related to sexual health. Oncology nurses are expected to fulfill a variety of activities such as information giving, symptom control, psychological care, and social support for the patient. Nurses have important duties as a counselor and guide in determining the factors affecting sexual functions of cancer patients, problems that may be experienced in sexual matters, and providing help to these individuals to get over these problems. [37, 50, 51]

In the light of the previous, the researchers conducted this study for evaluating the impact of a protocol of nursing intervention on sexual dysfunction and body image distress among women with cervical cancer. This aim was achieved through designing and application of protocol of nursing intervention on sexual dysfunction according to women's needs and evaluating the impact of the application of protocol of nursing intervention on sexual dysfunction among women with cervical cancer on sexual distress, functions, and body image.
The present study revealed the mean age of the study sample was $49.4 \pm 9.38$, and slightly more than one-third of women were in the $1^{\text {st }}$ degree when diagnosed with cervical cancer, more than one-third $(37.1 \%)$ received a combination of therapies. In the same line with our study findings; Ali et al., (2018) who studied "Sexual distress and sexual function in a sample of Iranian women with gynecologic cancers", describes the demographic profiles of the respondents that participants were an average of 43.6 years old and slightly more than one-third of the patients were in the first stage of cancer. And slightly less than onequarter of patients received a combination of therapies. This highlights the importance of screening women for early signs and symptoms of cervical cancer for better treatment outcomes and less long-term side effects from treatment. [52]

Regarding treatment type the current study showed that slightly more than one-third of women had received radiotherapy, chemotherapy, surgical operation, approximately all of them had a hysterectomy and less than half of the sample had diarrhea, hair loss, tiredness, and anemia. This finding is supported by Ahmed \& Hassan (2016). [51]

Concerning women's knowledge about cervical cancer; the results of the current study declare the women's total knowledge regarding cervical cancer. The pre-test of the present study revealed that the majority $(95.7 \%)$ of studied women had unsatisfactory knowledge. This lack of knowledge may be attributed to that around three-quarters $(70.0 \%)$ of the studied women had low levels of education and two-thirds were 
housewives. In the same line Mitiku \& Tefera, (2016) that studied "Knowledge about Cervical Cancer and Associated Factors among 15-49 Year Old Women in Dessie Town, Northeast Ethiopia" revealed that using the sum of all knowledge items determined that a total of slightly more than half of the participants had sufficient knowledge about cervical cancer at post-intervention. This may be due to the continuous education and motivation of women by the researcher through using the educational intervention package. [53]

However, there was a high statistical significant improvement in the women's knowledge about cervical cancer in which approximately the majority $(95.7 \%)$ of women had satisfactory knowledge at postintervention $(\mathrm{p}<0.001)$. This may be due to continuous education and encouragement among the studied women by using the protocol of nursing intervention. After the implementation of the protocol, the results indicated that there is a significant increase in women's knowledge. Moreover, the progression of a satisfactory score of women's grading and regression of unsatisfactory women's grading, after the implementation of the protocol compared to before, were observed associated with statistical differences. This improvement could be attributed to that all women of the sample were committed to the protocol. [54-55]

Regarding total scores of females' sexual functions index (FSFI); the current study findings showed that all $(100.0 \%$; Mean \pm SD $33.75 \pm 13.12)$ of women had sexual dysfunction at pre-intervention. This may be related to embarrassment, lack of access to information, low education about sex, and ignorance of communication about sexual concerns by the health care provider that are considered the main barriers to opening sexual discussion. Also, a large part of professionals in the health area is not found to be adequately prepared for the approach of sexual complaints. This can be related to the low knowledge, due to the lack of disciplines in curricular grades of graduation courses that approach human sexuality as not only limited to reproductive function.

However, half of the studied women (50.0\%; Mean \pm SD 55.13 \pm 8.31$)$ had sexual functioning at post-intervention and improved in items of FSFI. A highly statistically significant difference is observed in the total scores of FSFI among studied sample pre and post-intervention. This may be due to continuous education and support among the studied sample about sexual concerns with cervical cancer that provided great support and encouraging women to decrease immparesment talking about their sexual problems.

In agreement with our study result of Hassan et al., (2019) that studied "Comprehension of Dyspareunia and Related Anxiety among Northern Upper Egyptian women: Impact of Nursing Consultation Context Using PLISSIT Model" in Egypt revealed that there were statistically significant differences between pre and post-application of the PILLIST model $(\mathrm{P}<0.001)$ as regard to elements of female sexual function index (FSFI) including desire, arousal, orgasm, satisfaction, and pain. [37] Moreover, this is consistent with Rostamkhani et al., (2015) that studied "Addressing the sexual problems of Iranian women in a primary health care setting: A quasi-experimental study", they reported that significant improvement was found in FSFI sub-domain scores, including sexual desire, arousal, orgasm, satisfaction, lubrication and pain in the intervention group compared to the control group. [56]

Moreover, this result in the same line with Chun, (2011) that studied "Effectiveness of PLISSIT model sexual program on female sexual function for women with gynecologic cancer", and reported that the PLISSIT model sexual program is effective in increasing sexual function for women with gynecologic cancer. Nurses may contribute to improving women's sexual function by utilizing the program. Strategies to relieve sexual pain need to be considered for the greater effectiveness of the program. [57]
Regarding women's total sexual distress scores, this study revealed that the majority $(88.6 \%)$ of the studied women had sexual distress at preintervention; while all $(100.0 \%)$ of them had no sexual distress at postintervention. A highly statistically significant difference is observed among women in the total scores of the sexual distress scale. In-consistent with the current study findings Hee (2013) that study "Effect of PLISSIT Model Sexual Health Enhancement Program for Women with Gynecologic Cancer and Their Husbands" reported that post-intervention results showed significant differences between the groups for sexual function, sexual distress, and marital intimacy in the women and subjective happiness in the husbands. Results also indicate that the sexual health enhancement program is effective in improving sexual function, lowering sexual distress, increasing marital intimacy, and subjective happiness in women. [58]

In agreement with the current study findings Brotto et al., (2012) "A brief mindfulness-based cognitive-behavioral intervention improves sexual functioning versus wait-list control in women treated for gynecologic cancer" revealed that sexual treatment and education led to significant improvements in all domains of sexual response, and a trend towards significance for reducing sexual distress. Perception of genital arousal during an erotic film was also significantly increased following the intervention despite no change in physiologically-measured sexual arousal. [59] In disagreement with the current study findings Bakker, (2016) stated that participants' sexual distress was not significantly different over time. [60]

Regarding women's total body image scores, the present study indicated that the majority $(90 \%)$ of women had body image distress at preintervention; whereas after the intervention the majority $(97.1 \%)$ of them had good body image. A highly statistically significant difference is observed among women in the total scores of the body image scale. These findings were supported by Sewell \& Edwards (2015) that studied "Pelvic genital cancer: Body image and sexuality" who reported that treatment of gynecological cancer typically affects body parts associated with womanhood and femininity, causing considerable body changes that are likely to alter body image. After this treatment is completed, gynecological cancer patients are often left with the residual effects of surgery and/or various medical procedures, such as radiation and chemotherapy that may affect sexuality and couple satisfaction. [61]

In the same line Sook Ran, (2014) that conduct a study about "Comparison of Distress and Body Image according to the Stages of Cancer Survivorship in Gynecological Cancer Patients" found that the score of body image was significantly lower in the acute cancer survival stage than long-term cancer survival $(\mathrm{F}=3.69, \mathrm{p}=.026)$. Gynecological cancer patients in the acute cancer survival among the three stages will need nursing intervention to decrease distress and improve body image. [62-65]

In addition to Trachtenberg et al. , (2019) that studied "Feasibility and acceptability of i-Restoring Body Image after cancer; A pilot trial for female cancer survivors" revealed that the majority of their participants experienced significant reductions in body image distress and improvements in embodiment scores. These pre-intervention and postintervention changes were statistically significant. This may be related to the addressing of sexual concerns after cancer treatment in their nursing intervention and specifically body image related distress. [66]

Furthermore similar to the findings of El-Sayed \& Ahmed (2015) that conducted their study in Egypt represented body image scores pre and post-intervention among the studied women with breast cancer. It showed a statistically significant difference between body image scale level before and after application of the PLISSIT counseling model. This may be due to the high level of education among their studied women. [67] 
The present study revealed that there are statistically significant associations between women's total knowledge, body image scores, and sexual distress scores. Moreover, regression of all items of women's sexual distress scores post-intervention compared to pre-one. Additionally, progression and improvement of all items of women's body image scores post-intervention compared to pre-one. This may be attributed to the attending of the protocol sessions and the lecture and positive reinforcement or the long-term retention of knowledge, as well as wide verities of used educational used methods. [25, 40, 49, 68] As well as the distributed Arabic booklets, also, played a crucial role in attaining and retain knowledge about sexuality. Booklets are best used when they are brief, written in plain language, full of good pictures, and when they are used to back-up other forms of education. This is, in accordance, with Edgar Dale's or the NTL's Pyramid of Learning as cited by Masters as the pyramid illustrated that individuals can retain $10.0 \%$ of what they read and $20.0 \%$ of what they see and hear (audiovisual). The same author added that ones can retain $50.0 \%$ of what he learned by a discussion [69-78].

\section{Conclusions:}

Based on the finding of the present study, it can be concluded that: The findings of this study supported the research hypotheses that, there was a high statistically significant difference in the women's total score of knowledge about cervical cancer, the total score of female sexual function index, the total scores of female sexual distress scale and total scores of body image scale at pre and post-intervention after application of educational program. The study concluded that educational protocol, which provided to cervical cancer survivors' women, have a positive effect on their own knowledge, body image, and sexual function.

\section{Recommendations:}

In the light of the findings of the study, the following are suggested:

Disseminate the multidisciplinary collaboration approach for addressing sexual problems related to cervical cancer.

Preparing health classes for cervical cancer women regarding sexual dysfunction following cervical cancer.

Further research about women's perceptions and practices regarding sexual dysfunction with cervical cancer.

\section{References:}

1. Zagloul M., Naser E., Hassan H. (2020) Cervical Cancer Knowledge, Attitude, and Practices: Educational Program Management for Female Workers at Port Said University. International Journal of Studies in Nursing, 5(3): 1-16. doi:10.20849/ijsn.v5i3.776.

2. Habtu Y., Yohannes S., Laelago T. (2017) Knowledge, attitude and practice for cervical cancer prevention and control among women of childbearing age in Hossana Town, Hadiya zone, Southern Ethiopia: Communitybased cross-sectional study. PLoS ONE, 12(7): e0181415. doi: 10.1371/journal.pone.0181415

3. Atwa A., Hassan H., Ahmed S. (2019). The impact of a hospital-based awareness program on the knowledge of patients about breast cancer and cancer cervix. International Journal of Studies in Nursing, 4(1): 20-29. doi:10.20849/ijsn.v4i1.537.

4. Said S., Hassan H., Sarhan A. (2018) Effect of an Educational Intervention on Women's Knowledge and Attitude Regarding Cervical Cancer. American Journal of Nursing Research, 6(2): 59-66. doi: 10.12691/ajnr-6$2-4$.
5. Hassan H., Kamal H., Abd El Salam S., Ali R. (2021) Survivors from Cervical Cancer: Impact of an Educational Program on Self-Knowledge and body-Image. Public Health Open Access,; 5(2):1-7. DOI: 10.23880/phoa16000175

6. Qalawa, Sh., Eldeeb, A., \& Hassan, H. (2015) Young Adult Women's intention regarding breast and cervical cancer screening in Beni-Suef. Scientific Research Journal, 3(3): 11-24.

7. Ali R., Abd El Salam S., Kamal H., Hassan H. (2021) Women with Cervical Cancer: Impact of an Educational Program their Knowledge. Journal of Obstetrics Gynecology and Reproductive Sciences, 5(2): 1-8. DOI: 10.31579/2578-8965/063

8. Soleimani MA., Bahrami N., Yaghoobzadeh A., Parker A., Chan, YH. (2018) Sexual distress and sexual function in a sample of Iranian women with gynecologic cancers, European Journal of Oncology Nursing, 35: 47-53.

9. Hassan, H., Bayoumi, M., \& Atwa, A. (2016) Emotional Distress Associated with Gynecologic and Breast Cancer in Beni-Suef City. International Journal of Science and Research, 5(2): 1118-1129.

10. Brunault P., Champagne A.L., Huguet G., Suzanne I., Senon J.L., Body G., Rusch E., Magnin G., Voyer M., Réveillère C.(2016) Major depressive disorder, personality disorders, and coping strategies are independent risk factors for lower quality of life in nonmetastatic breast cancer patients. Psycho-Oncology, Wiley, 25 (5): 513-520.

11. Hassan H., Mohammed R., Ramadan S., Masaud H. (2021) Impact of an Educational Program on Sexual Issues among Cervical Cancer Survivors' Women in Northern Upper Egypt. Journal of Obstetrics Gynecology and Reproductive Sciences, 5(1): 1-16. DOI: 10.31579/ 2578$8965 / 061$

12. Júnior J., Dufloth R., Vale D, Lima M., Zeferino L.,(2017) Early Age at First Sexual Intercourse is Associated with Higher Prevalence of High-grade Squamous Intraepithelial Lesions (HSIL), Rev Bras Ginecol Obstet., 39(2):80-85. doi: 10.1055/s-00361597973.

13. Nady F., Said M., Youness E., Hassan H. (2018) Effect of Nursing Intervention Program on Quality of Life Improvement for Women Undergoing Gynecological and Breast Cancer Treatment. Assuit Scientific Nursing Journal, 6(15): 62-77.

14. Nady F., El-Sherbiny M., Youness E., Hassan H. (2018) Effectiveness of Quality of Life Planned Teaching Program on Women Undergoing Gynecologic Cancer Treatment. American Research Journal of Oncology, 1(1): $1-17$.

15. 15. Araujo F., Janaina L., Laurentino S., Vieira C.(2016) Quality of life and sexual function after cervical cancer: a cross-sectional study, online Brazilian Journal of Nursing, 15: 542-545.

16. Hofsjö A., Bergmark K., Blomgren B., Jahren H., Bohm N. (2018) Radiotherapy for cervical cancer - impact on the vaginal epithelium and sexual function. Acta Oncologica, 57(3):

338-345. doi.org/10.1080/0284186X.2017.1400684

17. Abd El Salam S., Hassan H., Kamal K., Ali R.(2021) Women's Sexual Dysfunction Associated with Cervical Cancer. Applied Science and Computer Mathematics, 2(1): 17-27. 
18. Hassan H., Ramadan S., Ali R., Kamal H. (2021) Sexual Issues among Cervical Cancer Survivors' Women in Northern Upper Egypt. Journal of Advanced Trends in Basic and Applied Science,; 1(1): 1-11

19. Masaud H., Hassan H., Mohammed R., Ramadan S. (2021) Women's Sexual Distress Associated with Cervical Cancer. Sumerianz Journal of Medical and Healthcare, 4(1): 28-34. doi.org/10.47752/sjmh.41.28.34

20. Hassan H., Nady F., Youns E., Zahran K. (2016) Call for Change Level of Knowledge, Awareness and Attitude to Follow A High Folate Diet Among Pregnant Women. IOSR Journal of Nursing and Health Science; 5(1): 93100. doi: 10.9790/1959-051293100

21. Hassan H. (2020) Quality of Life with Gestational Diabetes. American Research Journal of Public Health, 3(1): 1-4.

22. Hassan H. (2020) Fetal and neonatal complications of pregnancy induced hypertension. American Research Journal of Public Health, 3(1): 1-3. DOI: 10.21694/26393042.20003

23. Hassan H. (2020) Evidence-Based Practice in Midwifery and Maternity Nursing for Excellent Quality of Care Outcomes. American Journal of Nursing Research, 8(6): 606-607. doi: 10.12691/ajnr-8-6-3.

24. Hassan H., Mohamady Sh., Afifi H. (2016) Mobile Technology \& Nursing Education, Practice. Journal of Distance Learning and Open Learning, 4(6): 157-166.

25. Hassan H. (2019) Integrative Nursing Science in Women's Pre-conceptional Wellness. International Journal of Health and Biological Sciences, 2(1): 17-18.

26. Salvage, J., \& White, J. (2020). Our future is global: nursing leadershipand global health. RevistaLatinoamericana De Enfermagen, 28, e3339.

27. Hassan H., Zahran K., Youness E., Nady F. (2015) Pregnant Women's Awareness, Intention and Compliance regarding Folic Acid Usage for Prevention of Neural Tube Defects According to Health Belief Model in Beni-Suef City. Pyrex Journal of Nursing and Midwifery, 1(3): 1326.

28. Farg D. and Hassan H. (2019) Study Hyperemesis Graviderum Requiring Hospital Admission during Pregnancy: Effect of Nursing Implication on Its Progress. American Journal of Nursing Research, 7(3): 328-341. doi: 10.12691/ajnr-7-3-14.

29. Hassan H., Said S., Hassanine Sh. (2017) Disparities of Prevalence and Causes of Maternal Antenatal Anxiety among Primigravida Pregnant Women in Egypt. American Research Journal of Nursing, 3(1): 1-15. DOI:10.21694/2379-2922.17014

30. Mostafa H., Yousef F., Hassan H. (2018) Health Related Quality of Life Educational Interventions: Effect on Chronic Hepatitis C Patients'. Saudi Journal of Nursing and Health Care, 1(2): 56-67.

31. Hassan H. Kegels (2020) Exercises: A crucial issue during woman's lifespan. American Research Journal of Public Health, 3(1):1-5. DOI: 10.21694/2639-3042.20001

32. Hassan H. (2020) Human Breast Milk Bank: Valuable Issue for Working Mothers. American Research Journal of Public Health, 3(1): 1-3. DOI: 10.21694/2639-3042.20002

33. Sheha E., Hassan H., Genedy A. (2020) Hassanine Sh. Effect of educational program on mother's knowledge and practice regarding Hepatitis C Virus in rural areas. American Journal of Nursing Research, 8(3): 303-310. DOI: 10.12691/ajnr-8-3-1
34. Hassan H. (2020) Self-Care Practices for Women with Gestational Diabetes. EC Nursing and Healthcare, 2(12): 168-175.

35. Hassan H. (2020) Call to Improve Women's Awareness Regarding Emergency Contraception in Arab Societies. Journal of Human Physiology, 2(2): 6-10.

36. Evans D. (2013) Promoting sexual health and wellbeing: the role of the nurse. Nurs Stand., 28: 53-57.

37. Hassan H., Saber N., Sheha E. (2018) Comprehension of Dyspareunia and Related Anxiety among Northern Upper Egyptian women: Impact of Nursing Consultation Context Using PLISSIT Model. Nursing \& Care Open Access Journal, 6(1): 1-19. DOI: 10.15406/ncoaj.2019.06.00177

38. Chow K., Chan J. Cho K., Chan C. A (2016) review of psych educational interventions to improve sexual functioning, quality of life, and psychological outcomes in gynecological cancer patients. Cancer Nurs., 39(1): 20-31. doi: 10.1097/NCC.0000000000000234.

39. Ali R., Kamal H., Hassan H., Abd El Salam S. (2021) Impact of an Educational Program on Sexual Distress Associated With Cervical Cancer. Further Applied Healthcare, 1(1): 30-42

40. Nady F., Said M., Youness E., Hassan H. (2017) Impact of Tailored Educational Program of Quality of Life Improvement on Women Undergoing Breast Cancer Treatment at El-Minia Region, Egypt. American Research Journal of Gynaecology, 1(1): 1-17. doi:10.21694/25775928.17001

41. Mohamed A., Hassan H., Gamel W., Arafa A. (2019) Awareness about breast and cervical cancers among nursing students in Beni-Suef University. Journal of Nursing Education and Practice, 9(5): 44-51. doi.org/10.5430/jnep.v9n5p44

42. Hassan H. (2020) Early Stage Cervical Cancer: Survivorship and Fertility preservation. American Research Journal of Oncology, 2(1): 1-3.

43. Abd El Salam., Ali R., Hassan H., Kamal H. (2021) Outcome of an Educational Program on Body Image Distress Associated with Cervical Cancer. Journal of Advanced Trends in Basic and Applied Science; 1(1): 1220

44. Ramadan S., Hassan H., Masaud H., Mohammed R. (2021) Women's Body Image Distress Associated with Cervical Cancer. Journal of Obstetrics Gynecology and Reproductive Sciences; 5(3): 1-6. DOI: 10.31579/25788965/062

45. Hassan H., Masaud H., Mohammed R., Ramadan S. (2021) Self-Knowledge and Body Image among Cervical Cancer Survivors' Women in Northern Upper Egypt. Further Applied Healthcare, 1(1): 1-12

46. Anis T., Gheit S., Saied H., Al kherbash S. (2011) Arabic Translation of Female Sexual Function Index and Validation in an Egyptian Population. Journal of Sexual Medicine, 8(12):3370-3378. DOI: 10.1111/j.17436109.2011.02471.x

47. Derogatis L., Clayton A., D'Agostino D., Wunderlich G., Fu Y. (2008) Validation of the Female Sexual Distress Scale-Revised for Assessing Distress in Women with Hypoactive Sexual Desire Disorder. Journal of Sexual Medicine, 5(2):357-64: DOI: $10.1111 /$ j.17436109.2007.00672.x

48. Hopwood P., Fletcher I., Lee A., Ghazal S. (2011) A body image scale for use with cancer patients. European Journal 
of Cancer, 37(2):189-97. DOI: 10.1016/S09598049(00)00353-1

49. Ramadan E., Eldesokey A., Hassan H. (2020) Effect of an Educational Package on Knowledge, Practices, and Attitude of Premenopausal Women Regarding Sexuality. American Journal of Nursing Research, 8(5): 495-505. doi: 10.12691/ajnr-8-5-2.

50. Hassan H., Atwa A. (2017) Occupational Stress, Job Satisfaction and Cervical Screening Intention of Maternity Oncology Nurses, Medical Science \& Healthcare Practice, 1(1): 48-59. doi:10.22158/mshp.v1n1p48

51. Ahmed S. \& Hassan S. (2016) Application of PLISSIT Counseling Model for Women with Cervical Cancer Undergoing Treatment on Enhancing Sexuality, American Journal of Nursing Science, 5(3): 85-95

52. Ali M., Bahramia N. (2018), Yaghoobzadehb A., Parker A., Huak Y. Sexual distress and sexual function in a sample of Iranian women with gynecologic cancers, European Journal of Oncology Nursing, 35: 47-53.

53. Mitiku I., Tefera F. (2016) Knowledge about Cervical Cancer and Associated Factors among 15-49 Year Old Women in Dessie Town, Northeast Ethiopia. PLoS ONE, doi.org/10.1371/journal.pone.0163136

54. Hassan H. (2018) Effectiveness of a structured teaching program on anxiety and perception regarding toxoplasmosis among seropositive pregnant women in Northern Upper Egypt. Clinical Nursing Studies, 6(1): 119. doi.org/10.5430/cns.v6n1p1.

55. Hassanine Sh., Hassan H., Alkotb Z. (2017) Effect of Preventive Program on Progression of Osteoporosis among Female Patients over 40 years at El-Fayoum City. American Research Journal of Nursing, 3(1): 1-15. doi:10.21694/2379-2922.17012

56. Rostamkhani F., Jafari F, Ozgoli G., and Shakeri M. (2015) Addressing the sexual problems of Iranian women in a primary health care setting: A quasi-experimental study. Iranian Journal of Nursing and Midwifery Research, 20(1): 139-146.

57. Chun N. (2011) Effectiveness of PLISSIT model sexual program on female sexual function for women with gynecologic cancer, J Korean Acad Nurs., 41(4).

58. Hee J. (2013) Effect of PLISSIT Model Sexual Health Enhancement Program for Women with Gynecologic Cancer and Their Husbands. Journal of Korean Academy of Nursing,; 43(5): 681-689.

59. Brotto L., Erskine Y., Carey M., Ehlen T., Finlayson S., Heywood M., Kwon J., Stuart G., McAlpine J., Thomson S., Miller D. (2012) A brief mindfulness-based cognitive behavioral intervention improves sexual functioning versus wait-list control in women treated for gynecologic cancer. Gynecologic Oncology journal, 125 (2): 320-325.

60. Bakker R., Mens J., Groot H., Tuijnman C., Braat C., Hompus W., Poelman J., Laman M., Velema L., Kroon C., Doorn, H., Creutzberg C., ter M. (2017) A nurse-led sexual rehabilitation intervention after radiotherapy for gynecological cancer. Support Care Cancer, 25(3): 729737. doi: 10.1007/s00520-016-3453-2

61. 61. Sewell H., (1980) Edwards W. Pelvic Genital Cancer: Body Image and Sexuality, 2015, from Vaeth JM (ed): Body-Image, Self-Esteem, and Sexuality in Cancer Patients. 14th Annual San Francisco Cancer Symposium, San Francisco, Calif., 1979. Front Radiat Ther Oncol. Basel, Karger; 14: 35-41. doi.org/10.1159/000383861
62. Sook J., Ran H. (2014) Comparison of Distress and Body Image according to the Stages of Cancer Survivorship in Gynecological Cancer Patients, Asian Oncology Nursing, 14(1):15-22.

63. Pfaendler KS., Wenzel L., Mechanic MB., Penner KR. (2015) Cervical cancer survivorship: long-term quality of life and social support. Clin Ther., 37(1):39-48.

64. Prasongvej P., Nanthakomon T., Jaisin K., et al. (2017) Quality of Life in Cervical Cancer Survivors and Healthy Women: Thai Urban Population Study. Asian Pac J Cancer Prev., 18(2): 385-389.

65. Huang HY., Tsai WC., Chou WY., et al. (2017) Quality of life of breast and cervical cancer survivors. BMC Women's Health 17, 30.

66. Trachtenberg L., Wong J., Rennie H., McLeod D., Leung Y., Warner E., Jane M. (2019) Feasibility and acceptability of i-Restoring Body Image after Cancer (iReBIC): A pilot trial for female cancer survivors, PsychoOncology journal, 29(8).

67. El-Sayed N., Ahmed M. (2015) Effectiveness of Application of PLISSIT Counseling Model on Sexuality for Breast Cancer's Women Undergoing Treatment. American Journal of Nursing Science, (4): 218-230.

68. 68. Hassan H, Nasr E. (2017) Improving nurses' knowledge and skills regarding tocolytics for inhibiting preterm labor. Clinical Nursing Studies, 5(1): 1-12.

69. kamal H., Ali R., Abd El Salam S., Hassan H. (2021) Impact of an Educational Program on Sexual Dysfunction Associated With Cervical Cancer. American Journal of Nursing Research. 9(2)

70. Masters K. Edgar Dale's (2013) Pyramid of Learning in medical education: A literature review, Medical Teacher, 35(11): e1584-e1593.

71. Mohamed W., Hassan H. (2020) Effect of Instructional Supportive Guideline for Improving Women's Awareness towards Endometriosis. American Journal of Nursing Research, 8(1): 38-47. Doi:10.12691/ajnr-8-1-5.

72. Hassan H., Ahmed W., Mahmoud A. (2020) Impact of Tailored Educational Program on Primigravida Anxiety and Knowledge Regarding Minor Discomforts in Upper Egypt. International Journal of Studies in Nursing, 5(1): 118. doi:10.20849/ijsn.v5i1.698

73. Gamel W., Genedy A., Hassan H. (2020) Impact of Puerperal Sepsis Self-Care Nursing Guideline on Women's Knowledge and Practices. American Journal of Nursing Research; 8(2): 132-141. doi:10.12691/ajnr-8-2-1

74. Hassan H. (2019) The Impact of Evidence-Based Nursing as The Foundation for Professional Maternity Nursing Practices. Open Acc J Repro \& Sexual Disord., 2(2): 195$197 . \quad$ OAJRSD.MS.ID.000135. DOI: 10.32474/OAJRSD.2019.02.000135.

75. Mohammed F., Shahin M., Youness E., Hassan H. (2018) Survivorship in Women Undergoing Gynecological and Breast Cancer Treatment in Upper Egypt: The Impact of Quality of Life Improvement Educational Program". American Research Journal of Gynaecology, 2(1): 1-28. doi:10.21694/2577-5928.18001

76. Hassan H., Ali R., Abd El Salam S., Kamal H. (2021) Impact of an Educational Program on Sexual Dysfunction Associated With Cervical Cancer. Journal of Cancer Research and Treatment, 9(2)

77. Kamal H., Ali R., Abd El Salam S., Hassan H. (2021) SelfKnowledge among Women with Cervical Cancer. Journal 
of Cancer Research and Treatment, 9(1): 12-21. DOI: $10.12691 /$ jcrt-9-1-2

78. 78. Hassan H., Kamal H., Abd El Salam S., Ali R. (2021) Survivors from Cervical Cancer: Impact of an Educational
Program on Self-Knowledge and body-Image. Public Health Open Access, 5(2):1-7. DOI: 10.23880/phoa16000175 